\title{
Theory of solvent influence on reaction dynamics
}

\author{
A. A. Neufeld ${ }^{\text {a) }}$ \\ Abteilung für Spektroskopie und Photochemische Kinetik, Max-Planck-Institut für Biophysikalische Chemie, \\ Am Fassberg, D-37077 Göttingen, Germany
}

(Received 8 April 2004; accepted 9 February 2005; published online 28 April 2005)

\begin{abstract}
A generalization of the recently published quantum-classical approximation [A. A. Neufeld, J. Chem. Phys., 119, 2488 (2003)] for the purposes of reaction dynamics in condensed phase is presented. The obtained kinetic equations treat a solvent influence in a nonphenomenological way, account for the change of the free energy of the surrounding media, allow for different solvent dynamics in each reaction channel, and constitute a powerful framework for an accurate modeling of solvent effects, including ultrafast processes. The key features of the approach are its differential form, which considerably facilitates practical applications, and well defined wide applicability limits. The developed methodology fully accounts for an arbitrary long memory of the canonical bath and covers solvent-induced processes from a subpicosecond time scale. (C) 2005 American Institute of Physics. [DOI: 10.1063/1.1884515]
\end{abstract}

\section{INTRODUCTION}

A majority of reaction events in chemical and biological systems occur in condensed phase, which has stimulated extensive experimental and theoretical studies ${ }^{1}$ of the solvent influence on the course of reactions. This influence manifests itself in a number of ways. First, a solvent can chemically react with a solute, examples are proton transfer, ${ }^{2,3}$ substitution reactions, ${ }^{4,5}$ etc. Even a chemically inert solvent is able to form considerably stable complexes with molecules of interest (hydrogen bonding, ${ }^{3,5,6}$ solvation of ions ${ }^{7,8}$ ), and is capable of a substantial modification ${ }^{9,5}$ of the potential energy surfaces (PESs) of dissolved reactants. Second, a relatively strong interaction with solvent molecules commonly leads to fast decoherence of the quantum phase of transitions, thus mightily influencing a mechanism of an elementary process. In addition, the surrounding media alter the character of intermolecular and (low-frequency) intramolecular motions, changing them from dynamic into stochastic processes.

The complexity of the problem resulted in a formulation of theoretical approaches considerably simplified in comparison with those used for rarefied gases. In particular, intermolecular reactions in solution are usually considered in the approximation of separating out the relative motion of the reactants and their distant-dependent reaction probability. The latter is supposed to be mobility independent and is either calculated by appropriate theories ${ }^{10-12}$ or specified phenomenologically. ${ }^{13-15}$ This methodology turns out to be very fruitful when considering a wide class of diffusion assisted processes, ${ }^{15-20}$ and allows for a detailed study of the influence of reactant motion on the kinetics of both reversible and irreversible reactions.

There exist certainly several limitations for independent consideration of the reactivity and the mobility of dissolved molecules. First, at times shorter than a characteristic deco-

${ }^{a)}$ Electronic mail: aneufel@gwdg.de herence time the transitions between quantum levels proceed dynamically, and the description of an elementary process by means of transition probabilities is not adequate. Second, the efficiency of dephasing depends on many factors, while the solvent-induced damping of the reaction coordinate, driven by the high-frequency modes, is not as effective as the damping of the low-frequency ones. Thus, ultrafast reactions in condensed phase, proceeding in the (sub)picosecond time range, require a more accurate approach. Another rapidly growing field of research is the use of supercritical fluids as a reaction environment, ${ }^{21}$ where the density of the surrounding media can be smoothly varied from gas to liquid phase, thus gradually changing the mechanism of an elementary reaction from dynamic (rarefied gases) to stochastic (liquids), and some universal theoretical methodology is highly desirable to describe properly this interesting transitional region.

Existing approaches for an $a b$ initio simulation of reactions in condensed phase can be subdivided according to the treatment of solvent dynamics. A quantum-mechanical description of the solvent is inherent in rigorous semiclassical approaches, ${ }^{22}$ but it is feasible only for a few simple models. On the other hand, a classical description allows for a realistic modeling of the dynamics of the surrounding media by means of $a b$ initio molecular dynamics methods, ${ }^{23}$ and is an attractive alternative when quantum effects in the solvent dynamics are unimportant. Reacting subsystem can also be treated quantum mechanically or classically, but most of the reactions are intrinsically quantum mechanical (formation or breaking of chemical bonds, nonadiabatic transitions, etc.), thus leading to the quantum-classical treatment as the most suitable methodology for an adequate description of the reactions in condensed phase.

Despite the fact that the basic idea of the quantumclassical approach is rather simple, its practical implementations are very different and not equivalent to each other. In the most straightforward attempts to combine quantum and classical mechanics one adds quantum transitions directly to the classical trajectories, in the form of a mean-field 
approximation $^{22,24,25}$ (MFA) or a surface hopping ${ }^{26-28}$ (SH) scheme (or a combination ${ }^{29,30}$ of both). These approaches, however, have an essentially empirical flavor and unknown applicability limits. In a certain sense, MFA and $\mathrm{SH}$ are complementary schemes. The former treats the transitions dynamically, with a full account of quantum interference effects, but fails to describe properly a situation of considerably distinct channels of the reaction. In contrary, the $\mathrm{SH}$ scheme provides correct asymptotical solutions, but neglects the quantum interference effects (the transitions are treated as probabilistic jumps between PESs).

A quantum-classical Liouville equation ${ }^{31-36}$ (QCLE) for the reduced density matrix of the quantum subsystem seems to combine both the dynamical treatment of quantum transitions and the correct asymptotical solutions in the reaction channels. Various types of dynamics of the classical degrees of freedom can be used within the QCLE, which makes the approach an alluring one to study the reaction kinetics from gas to liquid state densities. Nevertheless, the QCLE also suffers from an internal inconsistency: it replaces the true cross coherence, which drives the energy flow between the subsystems, by the direct coherence inside the quantum subsystem, see Sec. III of Ref. 37 for more explanations. Among other things, it leads to a mean-field (or more precisely to a mean-potential) treatment of the transition phases, inherent in QCLE. Exactly this MFA feature of the QCLE is the source of unphysical predictions, ${ }^{38,39}$ when applied to nonadiabatic transitions.

A non-Markovian quantum-classical approximation ${ }^{40}$ (NQCA) is free from the aforementioned defects. It is based on a short coherence time of the interaction between the quantum subsystem and the canonical bath, has known applicability limits, and properly handles cross coherence between the subsystems. In the present paper we generalize the NQCA to extend its capabilities towards the treatment of reaction dynamics. The generalization consists of an assumption about the dynamics of the surrounding media, dependent on a state of the quantum subsystem, and is required to take into account the difference of interactions between the solute and the solvent in the distinct reaction channels, as well as the change of the free energy of the solvent in the course of reaction.

The outline of the paper is the following. Basic assumptions of the approach, small parameter, and partition of the Hamiltonian of the reacting system are presented in Sec. II. The assumptions made allow to separate out the reaction channels and to obtain the general non-Markovian master equations, see Sec. III. The corresponding memory kernels, describing various solvent-induces transitions, are then evaluated in a form suitable for the quantum-classical treatment, as outlined in Sec. IV and Appendix A. The latter is introduced in Sec. V. The kinetic equations of the quantumclassical approximation, which are of differential form, but fully account for the arbitrary long memory of the bath, are obtained in Sec. VI. Basic properties of these equations (positivity, detailed balance, etc.) are discussed in Sec. VII. The advantages of the developed approach in comparison with existing quantum-classical treatments are briefly dis- cussed in Sec. VIII. In the Conclusion we summarize the results and outline the direction of future work.

\section{BASIC ASSUMPTIONS}

To generalize the NQCA, we assume that the quantumdynamical subsystem (QDS) consists of groups of quantum states which interact with the thermal bath in different ways. This means that the Hamiltonian of the bath may depend on a quantum state of the QDS. Although the number of different groups may, in principle, be arbitrary, we assume that there are only the two groups of states, and denote the associated projectors as $\hat{P}$ and $\hat{C}$. Additionally, we require a commutativity of the projectors with the QDS Hamiltonian, and, therefore, the transitions between the distinct groups of quantum states are induced solely by the interaction with the solvent. In particular, when applied to nonadiabatic transitions, neither an adiabatic nor a diabatic representation is used. This is demanded to develop approximations based on a short coherence time of the QDS-bath interaction, and allows to take into account the solvent effects in the widest possible limits.

For the groups of discrete (bound) states the required projectors are constructed from the corresponding normalized eigenstates $\left|\psi_{k}\right\rangle$ and $\left|\phi_{j}\right\rangle$ as

$$
\hat{P}=\sum_{k}^{N_{p}} \hat{P}^{k}, \quad \hat{P}^{k}=\left|\psi_{k}\right\rangle\left\langle\psi_{k}\right|,
$$

and

$$
\hat{C}=\sum_{j}^{N_{c}} \hat{C}^{j}, \quad \hat{C}^{j}=\left|\phi_{j}\right\rangle\left\langle\phi_{j}\right|,
$$

where $\hat{P}^{k}$ and $\hat{C}^{j}$ are the projectors on the individual eigenstates, while $N_{p}$ and $N_{c}$ are the numbers of the states in the corresponding group. Although a continuous part of the spectrum of the QDS eigenstates can be included in the consideration by an appropriate modification of the projectors (they will contain integration over the continuous part), we content ourselves with a derivation assuming a discrete spectrum of eigenstates. Nonetheless, one can proceed to the corresponding limit in the resulting kinetic equations.

Then, we split the QDS Hamiltonian as

$$
\hat{H}=\hat{H}_{p}+\hat{H}_{c}, \quad \hat{H}_{p}=\hat{H} \hat{P}, \quad \hat{H}_{c}=\hat{H} \hat{C},
$$

since it is assumed to commutate with the projectors. In turn, the Hamiltonian of the bath involves interactions between solvent molecules and that part of the QDS-bath coupling, which does not induce transitions between the eigenstates of the QDS. It can be written as

$$
\hat{F}=\hat{F}_{p}+\hat{F}_{c}, \quad \hat{F}_{p}=\hat{F} \hat{P}, \quad \hat{F}_{c}=\hat{F} \hat{C},
$$

i.e., the bath dynamics is assumed to be group specific, but is independent on an individual state inside the group. If this is not the case, then the number of distinct groups of quantum states should be increased. We also note that these assumptions provide the commutativity of the bath Hamiltonian (4) with that of the QDS (3). 
The rest of the interactions enter the QDS-bath coupling Hamiltonian, which can be represented as

$$
\hat{W}=\hat{W}_{p}+\hat{W}_{c}+\hat{W}_{p c}+\hat{W}_{c p} .
$$

Here

$$
\hat{W}_{p}=\hat{P} \hat{W} \hat{P}, \quad \hat{W}_{c}=\hat{C} \hat{W} \hat{C},
$$

describe interactions between the group of quantum states and the bath, which induce transitions inside the given group, but not between distinct groups. In contrast,

$$
\hat{W}_{p c}=\hat{P} \hat{W} \hat{C}, \quad \hat{W}_{c p}=\hat{W}_{p c}^{\dagger}=\hat{C} \hat{W} \hat{P},
$$

where the dagger superscript denotes a Hermitian conjugate, describe the solvent-induced transitions between distinct groups of quantum states. The above partitioning of the Hamiltonians has a formal character, and can be done for an arbitrary system of interest. Nevertheless, we impose a restriction on the form of $\hat{W}_{p c}$ and $\hat{W}_{c p}$, and do not consider those solvent-induced transitions between distinct groups of quantum states, which are not accompanied by a corresponding change of the bath state (solvent-induced radiative transitions, ${ }^{41,42}$ for example).

The subsequent approximations are based on the smallness of the cross correlations between the QDS and the canonical bath, and require the matrix elements of the QDSbath coupling to satisfy ${ }^{40}$

$$
\frac{\left|\hat{W}_{i k}^{\alpha \beta}\right|}{\hbar} \tau_{b} \ll 1, \quad \alpha \neq \beta, \quad i \neq k, \quad \tau_{b}=\frac{\hbar}{\pi k_{B} T},
$$

where $\hbar$ is the Planck constant, the first multiplier $(W / \hbar)$ determines a rate of formation of the cross correlations, while $\tau_{b}$ is their characteristic lifetime ${ }^{40}$ due to an energy uncertainty of the bath degrees of freedom. Here and afterwards Latin and Greek letters label the QDS and the bath eigenstates, respectively. Thus, the criterion (8) actually means that the rate of cross-correlations formation is much slower than the rate of their decay. In the following Planck and Boltzmann constants are set to unity, i.e., $\hbar=1$ and $k_{B}$ $=1$, thus measuring interaction matrix elements and absolute temperature $T$ in frequency units.

The assumptions hold true for many solvent-induced reactions of interest and allow to separate out the reaction channels, as shown in the following section.

\section{GENERAL MASTER EQUATION AND SEPARATION OF REACTION CHANNELS}

Following Ref. 40, we transform the Liouville-von Neumann equation for the density matrix $\bar{\varrho}(t)$ of the total system (QDS + bath),

$$
\frac{d \bar{\varrho}(t)}{d t}=\delta(t) \varrho_{0}+i(\hat{\mathcal{H}}+\hat{\mathcal{F}}+\hat{\mathcal{W}}) \bar{\varrho}(t)
$$

where $\varrho_{0}$ is the initial density matrix, $\hat{\mathcal{H}}, \hat{\mathcal{F}}$, and $\hat{\mathcal{W}}$ are the commutator derived Liouville operators (superoperators) of the QDS, bath, and the QDS-bath interactions, respectively, into a fully equivalent integro-differential equation of the form

$$
\begin{aligned}
\frac{d \bar{\varrho}(t)}{d t}= & \left(\delta(t)+i \hat{\mathcal{W}} \hat{\mathcal{G}}_{0}(t)\right) \varrho_{0}+i(\hat{\mathcal{H}}+\hat{\mathcal{F}}) \bar{\varrho}(t) \\
& -\int_{-\infty}^{\infty} \hat{\mathcal{K}}(\tau) \bar{\varrho}(t-\tau) d \tau .
\end{aligned}
$$

Here the definition of the density matrix is extended for negative times using the stepwise Heaviside $\theta$ function as

$$
\bar{\varrho}(t)=\theta(t) \varrho(t),
$$

and the kernel

$$
\hat{\mathcal{K}}(\tau)=\hat{\mathcal{W}} \hat{\mathcal{G}}_{0}(\tau) \hat{\mathcal{W}}, \quad \hat{\mathcal{G}}_{0}(\tau)=\theta(\tau) e^{i \hat{\mathcal{H}} \tau} e^{i \hat{\mathcal{F}} \tau}
$$

Although the Nakajima-Zwanzig projection formalism ${ }^{43}$ provides an elegant way to eliminate the bath variables, it is not convenient for the problem under consideration. Indeed, there exist different types of correlations (or coherence, when using a quantum-mechanical terminology), those inside the QDS, the correlations between the bath states, and the QDS-bath cross correlations. Within the projection formalism, the latter two are actually moved into the kernel of corresponding master equation. Developing subsequent approximations to this kernel, which is defined via an exact solution to the problem, is not an easy task. On the other hand, Eq. (10) explicitly contains the QDS-bath cross correlations, which are subject to perturbative treatment, while the kernel on the right-hand side is of the simple form, given by Eq. (12).

The closed memory-type equations for the density matrices of the distinct groups of quantum states are obtained in a way similar to that described in Ref. 40, and require a weak QDS-bath coupling in the sense of the condition (8). In a first step, the total density matrix $\bar{\varrho}$ in the integrand on the righthand side of Eq. (10) can be replaced by

$$
\bar{\varrho}(t-\tau) \simeq \bar{\varrho}_{p}(t-\tau)+\bar{\varrho}_{c}(t-\tau),
$$

where

$$
\bar{\varrho}_{p}(t-\tau)=\hat{P} \bar{\varrho}(t-\tau) \hat{P}, \quad \bar{\varrho}_{c}(t-\tau)=\hat{C} \bar{\varrho}(t-\tau) \hat{C}
$$

are the density matrices projected on the corresponding subspaces. The approximation (13) neglects the correlations between $\bar{\varrho}_{p}$ and $\bar{\varrho}_{c}$, which can be of two types, the coherence inside the QDS and the cross coherence between the QDS and the bath. We postulate that the former type of coherence is absent at the initial instant of time, and, in accordance with the assumptions made, see text after Eq. (7), such direct coherence between distinct groups of quantum states will not appear afterwards as well. As regards the cross correlation between the QDS and the bath, it is small under condition (8), and, in general, is of second order in the QDS-bath coupling strength. On the other hand, the exact kernel in Eq. (10) is strictly proportional to the second power of the QDSbath interaction, and, therefore, the use of Eq. (13) in the integrand on the right-hand side of Eq. (10) retains the effect of the cross correlation between $\bar{\varrho}_{p}$ and $\bar{\varrho}_{c}$ to their lower order. 
Similarly, the criterion (8) allows to replace $\bar{\varrho}_{p}$ and $\bar{\varrho}_{c}$ in the integrand on the right-hand side of Eq. (10) by the direct products of the density matrices of the QDS and the bath, i.e.,

$$
\bar{\varrho}_{p}(t-\tau) \simeq \bar{\sigma}_{p}(t-\tau) \times \rho_{p}, \quad \bar{\varrho}_{c}(t-\tau) \simeq \bar{\sigma}_{c}(t-\tau) \times \rho_{c},
$$

where

$$
\bar{\sigma}_{p, c}(t-\tau)=\operatorname{Tr}_{\mathrm{b}} \bar{\varrho}_{p, c}(t-\tau)
$$

are the projected density matrices of the QDS, while

$$
\rho_{p, c}=\frac{1}{Z_{p, c}} e^{-\hat{F}_{p, c} / T}, \quad Z_{p, c}=\operatorname{Tr}_{\mathrm{b}}\left(e^{-\hat{F}_{p, c^{\prime}} T}\right)
$$

are the equilibrium density matrices of the corresponding bath, and $\operatorname{Tr}_{\mathrm{b}}$ denotes the trace over the bath variables. Thus, we assume that the transitions do not significantly change the equilibrium state of the bathes in the reaction channels. Again, the effect of the cross correlations between the QDS and the bath is retained to their lower order, despite the use of the factorized density matrices (15).

Note that the approximations (13) and (15) do not mean that the neglected off-diagonal elements between the QDS and the bath are small, they are only assumed to be out of phase. Situation is well illustrated by analogy with the discrete Fourier transform of exponentially decaying function, when the individual terms of expansion represent themselves an infinitely oscillating (nondecaying) functions with possibly large amplitudes. Nevertheless, after some time, their sum gives a negligibly small value due to the precise phase cancellation of the constituents.

Finally, condition (8) provides separation of the reaction channels. Indeed, operating on Eq. (10) from both sides by the corresponding projector, taking the trace over the bath variables, and restricting oneself to the lower order in the QDS-bath cross correlations, we obtain for the projected density matrices (16) the following set of the non-Markovian kinetic equations:

$$
\begin{aligned}
\frac{d \bar{\sigma}_{p}(t)}{d t}= & \delta(t) \sigma_{p 0}+\bar{\zeta}_{p}(t)-i\left[\hat{H}_{p}, \bar{\sigma}_{p}(t)\right] \\
& -\int_{-\infty}^{\infty} \hat{\mathcal{M}}_{p}(\tau) \bar{\sigma}_{p}(t-\tau) d \tau \\
& -\int_{-\infty}^{\infty} \hat{\mathcal{M}}_{1}(\tau) \bar{\sigma}_{p}(t-\tau) d \tau \\
& +\int_{-\infty}^{\infty} \hat{\mathcal{M}}_{2}(\tau) \bar{\sigma}_{c}(t-\tau) d \tau
\end{aligned}
$$

$$
\begin{aligned}
\frac{d \bar{\sigma}_{c}(t)}{d t}= & \delta(t) \sigma_{c 0}+\bar{\zeta}_{c}(t)-i\left[\hat{H}_{c}, \bar{\sigma}_{c}(t)\right] \\
& -\int_{-\infty}^{\infty} \hat{M}_{c}(\tau) \bar{\sigma}_{c}(t-\tau) d \tau \\
& +\int_{-\infty}^{\infty} \hat{\mathcal{M}}_{3}(\tau) \bar{\sigma}_{p}(t-\tau) d \tau \\
& -\int_{-\infty}^{\infty} \hat{\mathcal{M}}_{4}(\tau) \bar{\sigma}_{c}(t-\tau) d \tau,
\end{aligned}
$$

where $[\ldots, \ldots]$ denotes commutator, $\sigma_{p, c 0}$ are the initial conditions, $\bar{\zeta}_{p, c}(t)$ are the time-extended source terms due to the initial correlations, and the projected Hamiltonians $\hat{H}_{p, c}$ were defined earlier, see Eq. (3). The explicit expressions for the memory kernels will be given in the following section. Their particular feature is that $\hat{\mathcal{M}}_{p}(\tau)$ depends solely on $\hat{W}_{p}$, $\hat{\mathcal{M}}_{c}(\tau)$ depends solely on $\hat{W}_{c}$, while $\hat{\mathcal{M}}_{1-4}(\tau)$ contain only $\hat{W}_{p c}$ and $\hat{W}_{c p}$, see Eqs. (6) and (7). Mathematically, this is provided by the commutativity of the free propagator $\hat{\mathcal{G}}_{0}(\tau)$, defined in Eq. (12), with the projectors. As the result, the cross products of the form $\hat{W}_{p} \ldots \hat{W}_{p c}$, etc., are met only in combination with $\hat{P} \bar{\varrho}(t-\tau) \hat{C}$, or $\hat{C} \bar{\varrho}(t-\tau) \hat{P}$. The contributions of the latters are small and already proportional to the second power of the QDS-bath coupling, thus making the above cross products to be of higher order of smallness. In that way the reaction channels are separated out. Nonetheless, a specific interference between them enters the theory via memory-type dynamics.

To end this section, we note that the Hamiltonian renormalization of the form

$$
\hat{H}_{p} \rightarrow \hat{H}_{p}+\operatorname{Tr}_{\mathrm{b}} \hat{W}_{p} \rho_{p}, \quad \hat{W}_{p} \rightarrow \hat{W}_{p}-\operatorname{Tr}_{\mathrm{b}} \hat{W}_{p} \rho_{p},
$$

and

$$
\hat{H}_{c} \rightarrow \hat{H}_{c}+\operatorname{Tr}_{\mathrm{b}} \hat{W}_{c} \rho_{c}, \quad \hat{W}_{c} \rightarrow \hat{W}_{c}-\operatorname{Tr}_{\mathrm{b}} \hat{W}_{c} \rho_{c}
$$

is required ${ }^{40}$ for a fast decay of the time-extended source terms $\bar{\zeta}_{p, c}(t)$ on the time scale of $\tau_{b}$. In the following we assume that the renormalization was already done.

\section{REACTION KERNELS}

The memory kernels in Eqs. (18) and (19) describe various processes due to the interaction with the solvent. In particular, $\hat{\mathcal{M}}_{p, c}(\tau)$ involve the energy redistribution/relaxation inside the given group of quantum states, while $\hat{\mathcal{M}}_{1-4}(\tau)$ (reaction kernels) describe the solvent-induced transitions between the distinct groups. Since the former account for the processes in the uncoupled reaction channels, their structures can be taken from Ref. 40, where all variables (Hamiltonians, density matrices, etc.) should be treated as group specific and labeled by the corresponding subscript ( $p$ or $c$ ).

In the following we concentrate on the structure of reaction kernels. For the sake of convenience we introduce the following Liouville operators of the correlation functions: 


$$
\begin{aligned}
\hat{\mathcal{X}}_{1}(\tau) \bar{\sigma}_{p}(t-\tau)= & \frac{1}{Z_{p}} \operatorname{Tr}_{b}\left(e^{-i \hat{H}_{p} \tau} \bar{\sigma}_{p}(t-\tau)\right. \\
& \left.\times \hat{W}_{p c} e^{i \hat{H}_{c} \tau} \hat{W}_{c p}^{h}(\tau) e^{-\hat{F}_{p} / T}\right), \\
\hat{\mathcal{X}}_{2}(\tau) \bar{\sigma}_{c}(t-\tau)= & \frac{1}{Z_{c}} \operatorname{Tr}_{b}\left(e^{-i \hat{H}_{p} \tau} \hat{W}_{p c} \bar{\sigma}_{c}(t-\tau)\right. \\
& \left.\times e^{i \hat{H}_{c} \tau} e^{-\hat{F}_{c} / T} \hat{W}_{c p}^{h}(\tau)\right), \\
\hat{\mathcal{X}}_{3}(\tau) \bar{\sigma}_{p}(t-\tau)= & \frac{1}{Z_{p}} \operatorname{Tr}_{b}\left(\hat{W}_{c p}^{h}(\tau) e^{-\hat{F}_{p} / T} e^{-i \hat{H}_{p} \tau} \bar{\sigma}_{p}(t-\tau)\right. \\
& \times \hat{W}_{p c} e^{\left.i \hat{H}_{c} \tau\right)}, \\
\hat{\mathcal{X}}_{4}(\tau) \bar{\sigma}_{c}(t-\tau)= & \frac{1}{Z_{c}} \operatorname{Tr}_{b}\left(e^{-\hat{F}_{c} / T} \hat{W}_{c p}^{h}(\tau) e^{-i \hat{H}_{p} \tau} \hat{W}_{p c}\right. \\
& \left.\times \bar{\sigma}_{c}(t-\tau) e^{i \hat{H}_{c} \tau}\right),
\end{aligned}
$$

where

$$
\hat{W}_{c p}^{h}(\tau)=e^{i \hat{F}_{c} \tau} \hat{W}_{c p} e^{-i \hat{F}_{p} \tau}
$$

is the QDS-bath coupling in the Heisenberg representation with respect to the bath variables. Then, the explicit expressions for $\hat{\mathcal{M}}_{1-4}(\tau)$ take the form

$$
\begin{aligned}
& \hat{\mathcal{M}}_{1,3}(\tau) \bar{\sigma}_{p}(t-\tau)=\theta(\tau)\left(\hat{\mathcal{X}}_{1,3}(\tau) \bar{\sigma}_{p}(t-\tau)+\text { H.c. }\right) \\
& \hat{\mathcal{M}}_{2,4}(\tau) \bar{\sigma}_{c}(t-\tau)=\theta(\tau)\left(\hat{\mathcal{X}}_{2,4}(\tau) \bar{\sigma}_{c}(t-\tau)+\text { H.c. }\right)
\end{aligned}
$$

where H.c. denotes hermitian conjugate.

However, the presented form of the reaction kernels is not convenient when applied to reaction dynamics in condensed phase. First, a realistic modeling of the solvent is accessible only within a classical mechanics treatment, whereas the bath variables enter the correlation functions (22)-(25) in a way, which does not allow for the classical limit (due to time-ordered averages of bath operators). Second, the decay of some correlations between the reacting system and the canonical bath is essentially of quantummechanical nature, and is not correctly described by classical mechanics, so that the nonequilibrium energy flow between the subsystems has both "classical" and "quantum" components, see Ref. 44 for more details.

The above problems can systematically be resolved by appropriate transformation of the reaction kernels. Technically, it is necessary to pick out a generalized memory kernel describing the decay of the quantum correlations due to the energy uncertainty of the bath degrees of freedom. This procedure is more transparent for the bilinear QDS-bath couplings, see also Ref. 40. Although this assumption was used to simplify intermediate derivations only, it may, nevertheless, lead to some doubts ${ }^{45}$ about the validity of the obtained kinetic equations for the arbitrary (nonfactorized) form of the coupling. To remove this question, we give derivation for the general case.
Towards this end we introduce "symmetrized" correlation functions, which are obtained from Eqs. (22)-(25) just by formal replacement of $e^{-\hat{F}_{c} / T} \hat{W}_{c p}^{h}(\tau) / Z_{c}$ and $\hat{W}_{c p}^{h}(\tau) e^{-\hat{F}_{p} / T} / Z_{p}$ with

$$
\hat{V}_{c p}^{h}(\tau)=\frac{1}{2}\left(\frac{e^{-\hat{F}_{c} / T}}{Z_{c}} \hat{W}_{c p}^{h}(\tau)+\hat{W}_{c p}^{h}(\tau) \frac{e^{-\hat{F}_{p} / T}}{Z_{p}}\right) .
$$

The connection between Eqs. (22)-(25) and the corresponding symmetrized correlation functions is established in Appendix A. It decouples the statistical and the dynamical properties of the bath, and allows to take account of the quantum component of the energy flux between the QDS and the heat reservoir before proceeding to the classical limit for the bath degrees of freedom. The omission of this step leads to an incorrect treatment of the quantum correlations between the QDS and the classical bath, and, as a consequence, to the violation of the detailed balance.

Then, at times $t \gg \tau_{b}$ we derive the following set of the kinetic equations, see Appendix A:

$$
\begin{aligned}
\frac{d \bar{\sigma}_{p}(t)}{d t}= & \delta(t) \sigma_{p 0}-i\left[\hat{H}_{p}, \bar{\sigma}_{p}(t)\right]-\int_{-\infty}^{\infty} \hat{\mathcal{M}}_{p}(\tau) \bar{\sigma}_{p}(t-\tau) d \tau \\
& +\int_{-\infty}^{\infty} \theta(\tau) \operatorname{Tr}_{b}\left(e^{-i \hat{H}_{p} \tau} \bar{\gamma}_{p c}(t-\tau) e^{i \hat{H}_{c} \tau} i \hat{V}_{c p}^{h}(\tau)+\text { H.c. }\right) d \tau
\end{aligned}
$$

and

$$
\begin{array}{r}
\frac{d \bar{\sigma}_{c}(t)}{d t}=\delta(t) \sigma_{c 0}-i\left[\hat{H}_{c}, \bar{\sigma}_{c}(t)\right]-\int_{-\infty}^{\infty} \hat{\mathcal{M}}_{c}(\tau) \bar{\sigma}_{c}(t-\tau) d \tau \\
\quad-\int_{-\infty}^{\infty} \theta(\tau) \operatorname{Tr}_{b}\left(i \hat{V}_{c p}^{h}(\tau) e^{-i \hat{H}_{p} \tau} \bar{\gamma}_{p c}(t-\tau) e^{i \hat{H}_{c} \tau}+\text { H.c. }\right) d \tau,
\end{array}
$$

where

$$
\begin{aligned}
\bar{\gamma}_{p c}(t)= & -i\left(\hat{W}_{p c} \bar{\sigma}_{c}(t)-\bar{\sigma}_{p}(t) \hat{W}_{p c}\right) \\
& +\hat{U}_{p c} \bar{\sigma}_{c}(t)+\bar{\sigma}_{p}(t) \hat{U}_{p c}+\hat{Y}_{p c}\left(\frac{d \bar{\sigma}_{c}(t)}{d t}+i\left[\hat{H}_{c}, \bar{\sigma}_{c}(t)\right]\right) \\
& +\left(\frac{d \bar{\sigma}_{p}(t)}{d t}+i\left[\hat{H}_{p}, \bar{\sigma}_{p}(t)\right]\right) \hat{Y}_{p c}
\end{aligned}
$$

and we have assumed factorized initial conditions, which eliminate the time-extended source terms, ${ }^{40}$ i.e., $\bar{\zeta}_{p, c}(t)=0$. Here

$$
\hat{U}_{p c}=\int_{-\infty}^{\infty} f_{b}(\tau) e^{-i \epsilon_{p c} \tau} e^{-i \hat{H}_{p} \tau} \hat{W}_{p c} e^{i \hat{H}_{c} \tau} d \tau,
$$

and

$$
\hat{Y}_{p c}=-\int_{-\infty}^{\infty} \tau f_{b}(\tau) e^{-i \epsilon_{p c} \tau} e^{-i \hat{H}_{p} \tau} \hat{W}_{p c} e^{i \hat{H}_{c} \tau} d \tau,
$$

where the generalized memory kernel $f_{b}(\tau)$ describing the decay of quantum correlations is defined by Eq. (A21), and 


$$
\epsilon_{p c}=-T \ln \left(Z_{p} / Z_{c}\right)=-T\left(\ln Z_{p}-\ln Z_{c}\right)
$$

is the change of the free energy of the bath in the course of the reaction.

The derived from $\hat{W}_{p c}$ operators (33) and (34), see also Eqs. (A27) and (A28), are responsible for the detailed balance $\left(\hat{U}_{p c}\right)$, and for the positivity of the density matrices within the applicability limits of the approach ("slippage" operator $\hat{Y}_{p c}$ ). The value of $\epsilon_{p c}$, which enters the detailed balance condition together with the QDS transition frequencies, is formally expressed in terms of the statistical sums of the baths $Z_{p, c}$. From the practical point of view, the change of the free energy is frequently available from experimental data, and can also be extracted from $a b$ initio simulations. ${ }^{46,47}$ A specific approach to the determination of this quantity will be outlined on practical applications of the method.

Nevertheless, the bath in Eqs. (30)-(32) is still treated quantum mechanically, and the system-bath couplings have full dimensionality in the subspaces of the QDS and of the bath, which embarrasses practical use of the method. Furthermore, realistic simulations of solvent dynamics are provided by molecular dynamics methods, based on classical mechanics. The desired transition to the classical mechanics treatment of the bath is described in the following section.

\section{QUANTUM-CLASSICAL APPROXIMATION}

Up to now, the two approximations were employed. The first one restricts the strength of the system-bath couplings to the values (8), which has allowed to separate out the reaction channels and to obtain the closed kinetic equations for the density matrices of the QDS. The second approximation is based on the short lifetime of quantum correlations and was used to integrate out of the quantum component of the nonequilibrium energy flux between the QDS and the thermal bath. The latter becomes dominant if the condition (8) is not fulfilled (see Ref. 44 for more details) and, therefore, the classical mechanics treatment of the bath seems to be problematic in this limit.

A quantum-classical approximation is introduced as usual by replacing the correlation functions with the appropriate classical analogs. Although a search of such analogs is always based more on intuition, than on rigorous derivation, we give some arguments explaining our choice. First, the reaction kernel in Eq. (30) contains the retarded correlation function of the form [the correlation function in Eq. (31) differs by the ordering of the couplings]

$$
\begin{aligned}
\hat{\mathcal{J}}_{\mathrm{ret}}(\tau)= & \frac{\theta(\tau)}{2} \operatorname{Tr}_{\mathrm{b}}\left\{\hat { W } _ { p c } \ldots e ^ { i \hat { F } _ { c } \tau } \left(\frac{e^{-\hat{F}_{c} / T}}{Z_{c}} \hat{W}_{c p}\right.\right. \\
& \left.\left.+\hat{W}_{c p} \frac{e^{-\hat{F}_{p} / T}}{Z_{p}}\right) e^{-i \hat{F}_{p} \tau}\right\}
\end{aligned}
$$

where the dots denote some operators of the QDS. Strictly speaking, in the classical limit the QDS-bath coupling $\hat{W}_{p c}$ depends on the phase space coordinates of both baths, i.e.,

$$
\hat{W}_{p c} \underset{\text { limit }}{\stackrel{\text { classical }}{\rightarrow}} \hat{W}_{p c}\left(q_{p}, q_{c}\right),
$$

where the operator on the right has matrix elements only in the QDS subspace. However, the classical limit for the heat reservoir implies that the processes of quantum nature do not play significant role in its dynamics. Therefore, we assume that the QDS-bath interaction does not lead to the tunneling inside the bath, i.e.,

$$
\hat{W}_{p c}\left(q_{p}, q_{c}\right)=\hat{W}_{p c}\left(q_{p}\right) \delta\left(q_{p}-q_{c}\right)=\hat{W}_{p c}\left(q_{c}\right) \delta\left(q_{p}-q_{c}\right),
$$

and one can simply write

$$
\hat{W}_{p c} \underset{\text { limit }}{\stackrel{\text { classical }}{\longrightarrow}} \hat{W}_{p c}(q),
$$

where $q$ is the set of the phase space coordinates (either $q_{p}$ or $\left.q_{c}\right)$.

Then, it is readily seen that Eq. (36) contains averaging over different baths, being in the equilibrium and characterized by the corresponding bath Hamiltonian. It is, therefore, logical to replace the quantum averages by the sum of the dynamical averages over the sets of equilibrium classical trajectories, belonging to different baths, which gives

$$
\hat{\mathcal{J}}_{\text {ret }}(\tau)=\iint \hat{W}_{p c}(q) \bar{\varphi}\left(q, q^{\prime} ; \tau\right) \ldots \hat{W}_{c p}\left(q^{\prime}\right) d q d q^{\prime},
$$

where

$$
\begin{aligned}
\bar{\varphi}\left(q, q^{\prime} ; \tau\right)= & \left(\bar{\varphi}^{(p)}\left(q, q^{\prime} ; \tau\right) \psi^{(p)}\left(q^{\prime}\right)\right. \\
& \left.+\bar{\varphi}^{(c)}\left(q, q^{\prime} ; \tau\right) \psi^{(c)}\left(q^{\prime}\right)\right) / 2,
\end{aligned}
$$

and the generalized two-dimensional densities obey

$$
\begin{aligned}
\frac{\partial}{\partial \tau} \bar{\varphi}^{(p, c)}\left(q, q^{\prime} ; \tau\right)= & \delta(\tau) \delta\left(q-q^{\prime}\right)-i \Omega_{p c}(q) \bar{\varphi}^{(p, c)}\left(q, q^{\prime} ; \tau\right) \\
& +\mathcal{L}_{q}^{(p, c)} \bar{\varphi}^{(p, c)}\left(q, q^{\prime} ; \tau\right) .
\end{aligned}
$$

Here

$$
\psi^{(p, c)}(q)=e^{-F_{p, c}(q) / T} / \mathcal{Z}_{p, c}, \quad \mathcal{Z}_{p, c}=\int e^{-F_{p, c}(q) / T} d q,
$$

is the normalized equilibrium Boltzmann distribution (static contour) for the corresponding reaction channel, and

$$
\Omega_{p c}(q)=F_{p}(q)-F_{c}(q),
$$

where $F_{p, c}(q)$ are the Hamiltonian functions of the baths, and $\mathcal{L}_{q}^{(p, c)}$ are the linear functional operators describing evolution in their phase spaces.

The assumed analog (40) of the quantum correlation function (36) differs from those used in previous works ${ }^{40,37}$ in two aspects. First, it is complex due to the second term on the right-hand side of Eq. (42), which is proportional to $1 / \hbar$ in nonreduced units and, therefore, describes a quantum effect of the bath. The origin of this term is explained by the fact that the energy gap for the transition consists both of the energy splitting between the QDS eigenstates and of the change of the bath energy provided by the difference (44) of 
the bath Hamiltonian functions in the different reaction channels. Second, the correlation function (40) is an equally weighted sum of the averages over the distinct baths. Of course, substituting $F_{p}(q)=F_{c}(q)$ and $\mathcal{L}_{q}^{(p)}=\mathcal{L}_{q}^{(c)}$ into Eqs. (41)-(44), we arrive at the conventional definition of the classical correlation function.

It is important to note that a mean-potential bath dynamics for the transition matrix element, inherent in QCLE, would require in Eq. (36) an averaging over equilibrium bath state $\sim e^{-\left(\hat{F}_{p}+\hat{F}_{c}\right) / 2 T}$. This is obviously not the case, and, therefore, the corresponding feature of the QCLE is an artefact.

The necessity to solve memory-type kinetic equations considerably complicates practical applications of the developed quantum-classical approach, and, therefore, we transform them into a fully equivalent system of differential equations. This step does not involve any additional approximations, and is described in the following section.

\section{DIFFERENTIAL FORM OF THE QUANTUM- CLASSICAL APPROXIMATION}

By introducing several auxiliary matrices, the memorytype equations (30) and (31), where the quantum correlation function was replaced by the quantum-classical analog (40), can be reduced to the following set of differential equations, written using the conventional (nongeneralized) functions:

$$
\begin{aligned}
\frac{d \sigma_{p}(t)}{d t}= & -i\left[\hat{H}_{p}, \sigma_{p}(t)\right]-i \int\left[\hat{W}_{p}(q), \eta_{p}(q, t)\right] d q \\
& +\int\left(\chi_{p c}(q, t) i \hat{W}_{c p}(q)+\text { H.c. }\right) d q,
\end{aligned}
$$

and

$$
\begin{aligned}
\frac{d \sigma_{c}(t)}{d t}= & -i\left[\hat{H}_{c}, \sigma_{c}(t)\right]-i \int\left[\hat{W}_{c}(q), \eta_{c}(q, t)\right] d q \\
& -\int\left(i \hat{W}_{c p}(q) \chi_{p c}(q, t)+\text { H.c. }\right) d q
\end{aligned}
$$

where the auxiliary matrix

$$
\chi_{p c}(q, t)=\frac{1}{2}\left(\chi_{p c}^{(p)}(q, t)+\chi_{p c}^{(c)}(q, t)\right)
$$

is an equally weighted sum of two other ones, which differ only by the associated bath dynamics, indicated by the superscript, i.e.,

$$
\begin{aligned}
\frac{\partial \chi_{p c}^{(p)}(q, t)}{\partial t}= & i\left(\chi_{p c}^{(p)}(q, t) \hat{H}_{c}-\hat{H}_{p} \chi_{p c}^{(p)}(q, t)\right)+\gamma_{p c}(q, t) \\
& \times \psi^{(p)}(q)-i \Omega_{p c}(q) \chi_{p c}^{(p)}(q, t)+\mathcal{L}_{q}^{(p)} \chi_{p c}^{(p)}(q, t),
\end{aligned}
$$

while the equation for $\chi_{p c}^{(c)}(q, t)$ is obtained from Eq. (48) by replacing superscript " $p$ " with "c." Here also

$$
\begin{aligned}
\gamma_{p c}(q, t)= & -i\left(\hat{W}_{p c}(q) \sigma_{c}(t)-\sigma_{p}(t) \hat{W}_{p c}(q)\right)+\hat{U}_{p c}(q) \sigma_{c}(t) \\
& +\sigma_{p}(t) \hat{U}_{p c}(q)+\hat{Y}_{p c}(q)\left(\frac{d \sigma_{c}(t)}{d t}+i\left[\hat{H}_{c}, \sigma_{c}(t)\right]\right) \\
& +\left(\frac{d \sigma_{p}(t)}{d t}+i\left[\hat{H}_{p}, \sigma_{p}(t)\right]\right) \hat{Y}_{p c}(q),
\end{aligned}
$$

where the expressions for $\hat{U}_{p c}(q)$ and $\hat{Y}_{p c}(q)$ are obtained from Eqs. (33) and (34), or from Eqs. (A27) and (A28) by formal replacement of $\hat{W}_{p c}$ with $\hat{W}_{p c}(q)$.

The other auxiliary matrices satisfy ${ }^{40}$

$$
\begin{aligned}
\frac{\partial \eta_{p}(q, t)}{\partial t}= & -i\left[\hat{H}_{p}, \eta_{p}(q, t)\right]+\gamma_{p}(q, t) \psi^{(p)}(q) \\
& +\mathcal{L}_{q}^{(p)} \eta_{p}(q, t),
\end{aligned}
$$

where

$$
\begin{aligned}
\gamma_{p}(q, t)= & -i\left[\hat{W}_{p}(q), \sigma_{p}(t)\right]+\left[\hat{U}_{p}(q), \sigma_{p}(t)\right]_{+} \\
& +\left[\hat{Y}_{p}(q), \frac{d \sigma_{p}(t)}{d t}+i\left[\hat{H}_{p}, \sigma_{p}(t)\right]\right]_{+} .
\end{aligned}
$$

Here $[\ldots, \ldots]_{+}$denotes anticommutator, and

$$
\begin{aligned}
& \hat{U}_{p}(q)=\int_{-\infty}^{\infty} f_{b}(\tau) e^{-i \epsilon_{p c} \tau} e^{-i \hat{H}_{p} \tau} \hat{W}_{p}(q) e^{i \hat{H}_{c} \tau} d \tau, \\
& \hat{Y}_{p}(q)=-\int_{-\infty}^{\infty} \tau f_{b}(\tau) e^{-i \epsilon_{p c} \tau} e^{-i \hat{H}_{p} \tau} \hat{W}_{p}(q) e^{i \hat{H}_{c} \tau} d \tau,
\end{aligned}
$$

while replacing index $p$ with $c$ in Eqs. (50)-(53) one gets the equation for $\eta_{c}(q, t)$.

In the above equations $\hat{W}_{p}(q), \hat{W}_{c}(q)$, and $\hat{W}_{p c}(q)$ are the quantum operators of the QDS, parametrically dependent on the phase space coordinates of the baths. They have the dimensionality of $N_{p} \times N_{p}, N_{c} \times N_{c}$, and $N_{p} \times N_{c}$, respectively, and the dimensionality of the auxiliary functions $\eta_{p}(q, t)$, $\eta_{c}(q, t)$, and $\chi_{p c}(q, t)$ coincide with the dimensionality of the corresponding QDS-bath coupling. However, we warn against direct use of such quantum-classical form of the QDS-bath interaction in the framework of Schrödinger or Liouville equations, as this leads to the internal inconsistency. ${ }^{37}$ In contrast, before passing to the quantumclassical approximation, we have constrained several important features such as the proper treatment of the QDS-bath cross coherence, the detailed balance, etc., thus avoiding possible problems. The equivalence of Eqs. (45) and (46) and Eqs. (30) and (31) in the case of the quantum-classical definition of the correlation function given by Eq. (40) is proved following a general scheme used in Ref. 40.

The initial conditions for the density matrices,

$$
\sigma_{p}(0)=\sigma_{p 0}, \quad \sigma_{c}(0)=\sigma_{c 0},
$$

specify the starting distributions in the reaction channels, while the nonzero initial values for the auxiliary matrices,

$$
\chi_{p c}^{(p, c)}(q, 0)=\left(\hat{Y}_{p c}(q) \sigma_{c 0}+\sigma_{p 0} \hat{Y}_{p c}(q)\right) \psi^{(p, c)}(q),
$$

and

$$
\begin{aligned}
& \eta_{p}(q, 0)=\left[\hat{Y}_{p}(q), \sigma_{p 0}\right]_{+} \psi^{(p)}(q), \\
& \eta_{c}(q, 0)=\left[\hat{Y}_{c}(q), \sigma_{c 0}\right]_{+} \psi^{(c)}(q)
\end{aligned}
$$

account for the specific QDS-bath correlations created during the time scale of $\tau_{b}$, which is important to preserve the positivity of the density matrices within the applicability limits of 
the approach, see Ref. 40 for more details. [Equations (49) and (51) contain the time derivatives on the right-hand sides, which give the corresponding initial values for the auxiliary functions, when the generalized functions of time are replaced by the nongeneralized ones.]

An important feature of the obtained kinetic equations is that the explicit construction of the Liouville operators is avoided, and the propagation in time requires only matrix multiplications. For the Redfield theory the required decomposition of the relaxation tensor was suggested in Ref. 48, which greatly reduces computational cost, and, consequently, extends the class of tractable systems towards multilevel ones. On the other hand, our method has considerably wider applicability limits than that of the Redfield theory, and together with a slightly modified molecular dynamics approach, used in Ref. 37 to solve partial differential equations, may offer a similar computation performance.

\section{BASIC PROPERTIES OF KINETIC EQUATIONS}

The kinetic equations (45) and (46), obtained in the quantum-classical approximation describe the solventinduced transitions in the QDS consisting of two distinct groups of quantum states. These equations are valid at times $t \gg \tau_{b}$, fully account for arbitrarily long memory of the solvent degrees of freedom, and preserve the basic properties of the density matrices. First, the Hermiticity of the density matrices $\sigma_{p, c}$ is intact, as it can be seen by taking the Hermitian conjugate of Eqs. (45) and (46). Second, the sum of the traces of $\sigma_{p}$ and $\sigma_{c}$ is conserved and equal to the unity, i.e.,

$$
\operatorname{Tr}_{p} \sigma_{p}+\operatorname{Tr}_{c} \sigma_{c}=1,
$$

which is proved by taking the time derivative of Eq. (57) and substituting the corresponding expressions from Eqs. (45) and (46). Then we use the identity

$\operatorname{Tr}_{p}\left(\chi_{p c}(q, t) i \hat{W}_{c p}(q)+\right.$ H.c. $)=\operatorname{Tr}_{c}\left(i \hat{W}_{c p}(q) \chi_{p c}(q, t)+\right.$ H.c. $)$,

where $\operatorname{Tr}_{p, c}$ denotes the trace over the quantum states of the corresponding group, which follows from the cyclic permutation under the trace operation. It is seen that the terms describing the transitions between distinct groups of quantum states cancel each other, while the traces of the other constituents are zero, as shown in Ref. 40. Third, the positivity of the density matrices is maintained within the applicability limits (8), which is provided by the proper account for the finite duration of the characteristic time $\tau_{b}$. As a result, both the initial conditions and the relaxation operators are modified, and the role of such a modification for the conservation of the positivity in the course of the time evolution was well demonstrated. ${ }^{40}$ Nonetheless, the mathematical proof of the positivity can hardly be done in the general case, and we validate our conclusion numerically for several particular cases elsewhere.

Additionally, Eqs. (45) and (46) satisfy detailed balance both inside each reaction channel and between them. Indeed, the stationary solutions of Eqs. (45) and (46) are of the form

$$
\sigma_{p}=\alpha_{p} e^{-\hat{H}_{p} / T}, \quad \sigma_{c}=\alpha_{c} e^{-\hat{H}_{c} / T},
$$

where $\alpha_{p}$ and $\alpha_{c}$ are some non-negative constants, determining the probability of being in the corresponding reaction channel (they involve the statistical sums over the quantum states as well). Equilibrium between the reaction channels requires, see Appendix B,

$$
\alpha_{p} / \alpha_{c}=e^{-\epsilon_{p c} / T}
$$

where $\epsilon_{p c}$ is the change of free energy of the reaction (35). Equation (60) along with the normalization condition (57) allow to determine the coefficients $\alpha_{p, c}$ provided that the solvent-induced reaction lasts for a sufficiently long time (this may not be the case if reactants can be infinitely separated by translational diffusion, for example). Thus, Eqs. (59) and (60) satisfy the equilibrium conditions, and account for the change of free energy of the surrounding media in the course of reaction.

\section{DISCUSSION}

The developed method has wide and well established applicability limits, a time resolution of tens of femtoseconds, allows moderate strength of the QDS-bath couplings (up to $5 \times 10^{13} \mathrm{rad} / \mathrm{s}$ at $T=300 \mathrm{~K}$ ), and provides a powerful framework for accurate modeling the solvent influence on the course of the reaction. On the other hand, there is a variety of other quantum-classical approaches to reaction dynamics in condensed phase, and the development of an alternative method is justified only if it has considerable advantages over the existing ones. ${ }^{45}$ The required comparison would be simple if all other approaches also had explicitly declared applicability limits in terms of characteristic parameters. In that case it would be sufficient to compare the applicability limits to reveal differences between the methods.

Thus, it is relatively easy to compare our novel approach with the standard weak coupling limit and with Redfield theory. A principal advantage is that our method has femtosecond time resolution and is capable of describing nearresonance transitions in a deeply non-Markovian regime, which is a characteristic feature of many ultrafast reactions.

The NQCA is also different from the (generalized) Langevin equation approach, which essentially relies upon the bilinear coupling to the bath. In our case, bilinear coupling is not a prerequisite, and, therefore, our methodology is valid beyond this restriction. Besides, the derived kinetic equations incorporate the canonical equilibrium directly, and the fluctuation dissipation theorem, which provides a link between the fluctuations and the linear response, is not used at all.

It is difficult, however, to directly compare NQCA with other quantum-classical treatments. The comparison with hybrid schemes calls for extensive benchmarking, which goes beyond the scope of this paper. We only note here that the method is free of the intrinsic drawbacks inherent in hybrid approaches. 


\section{CONCLUSION}

We presented the generalization of the recently suggested non-Markovian quantum-classical approximation ${ }^{40}$ for the purposes of reaction dynamics in condensed phase. Toward this end we assumed that the quantum subsystem consists of two groups of quantum states, which interact with the surrounding media in different ways. This is typical for charge transfer reactions, recombination and dissociation, etc., and takes several important factors, such as the change of the free energy of the solvent in the course of reaction, and the peculiar bath dynamics in each reaction channel. The obtained kinetic equations can easily be modified to account for a larger number of distinct groups of quantum states, and constitute the basis for an accurate modeling of reaction dynamics in condensed phase, including ultrafast processes, and with a nonphenomenological treatment of the elementary reaction.

An essential feature of the kinetic equations is their differential form, which considerably facilitates practical applications. Nevertheless, the approach fully accounts for an arbitrarily long memory of the bath degrees of freedom, and properly takes into account the evolution during the initial time period $\left(\sim \hbar / k_{B} T\right.$, when the differential description is not valid) via an appropriate modification of the initial conditions and of the relaxation operators. This is particularly important to preserve the positivity of the density matrices within the applicability limits of the approach. Another distinctive feature of the method is the direct incorporation of the canonical equilibrium into the kinetic equations, which also account for the change of the free energy of the surrounding media.

The developed approach deals exclusively with the solvent impact on the reaction dynamics. In some cases, when the reaction of interest cannot proceed without the surrounding media (solvent-induced nonadiabatic transitions, recombination, etc.), solvent effects play the decisive role. In other situations the interaction with the bath molecules exerts a complementary influence, but is capable of considerably changing the reaction kinetics and the mechanism of the elementary reaction. In our formulation we assume that the solution to the problem is known for the isolated quantum subsystem, thus working with its eigenstates. In particular, when applied to nonadiabatic transitions, neither adiabatic nor diabatic representations are used. This is required for the use of the declared small parameter, and allows to take into account solvent effects in the widest possible limits. Additional approximations to the dynamics of quantum subsystem can be included in the methodology presented, provided that the subsidiary small parameters exist for the system under consideration.

State-specific dynamics of the bath is frequently used within the framework of the quantum-classical Liouville equation. The crucial point of this approach is a meanpotential treatment of the transition phase, which is a source of unphysical behavior in some situations. In contrast, our approach asserts a different recipe, which we would call a mean-evolution one. In other words, averaging concerns not the bath potentials, but the auxiliary matrices (memory), calculated for the true bath dynamics in each reaction channel.

In the present paper the novel non-Markovian approach to the reaction dynamics in condensed phase was formulated in the most general way, while its application to particular problems of interest requires additional efforts. We start with a simple case of nonadiabatic transitions between two localized modes, when the equilibration in their phase spaces is much faster than the characteristic time scale of the reaction (see the accompanying paper). Then, the reaction coordinates may be considered as belonging to the canonical bath, while the model is a direct counterpart of the nonadiabatic RRKM approach, and is of practical interest. In addition, this simple model allows for a detailed check of the new ingredients (change of free energy of the surrounding media in the course of reaction and the quantum-mechanical contribution of the bath dynamics) of the generalized treatment. In the following we plan to apply the presented methodology to solvent-induced nonadiabatic transitions for the more general case, and to recombination reactions in the condensed phase, as the new approach is perfectly suited to describe solventassisted transitions between the states of scattering of the reacting pair and its bound states. This work is now in progress.

\section{ACKNOWLEDGMENTS}

The author is grateful to Professor Jörg Schroeder for helpful discussions and to Professor Jürgen Troe for his continuous support. This work was partially supported by the Deutsche Forschungsgemeinschaft (DFG).

\section{APPENDIX A: TRANSFORMATION OF REACTION KERNELS}

The connection between the reaction kernels Eqs. (22)-(25) and their symmetrized counterparts, see Eq. (29) and the text above it, is established using the two-sided Fourier transform

$$
\widetilde{a}(\omega)=\int_{-\infty}^{\infty} a(t) e^{-i \omega t} d t,
$$

where $a(t)$ is an arbitrary function, defined on the entire time axis.

First of all, we represent the operator exponents as

$$
e^{ \pm i \hat{H}_{p} \tau} \equiv \sum_{k}^{N_{p}} e^{ \pm i E_{p}^{k} \tau} \hat{P}^{k}, \quad e^{ \pm i \hat{H}_{c} \tau} \equiv \sum_{j}^{N_{c}} e^{ \pm i E_{c}^{j} \tau} \hat{C}^{j},
$$

where the projectors on the individual eigenstates $\hat{P}^{k}$ and $\hat{C}^{j}$ were defined in Eqs. (1) and (2), $E_{p}^{k}$ and $E_{c}^{j}$ are the corresponding eigenvalues of $\hat{H}_{p}$ and $\hat{H}_{c}$, and fix the QDS transition frequency

$$
\omega_{k j} \equiv E_{p}^{k}-E_{c}^{j}
$$

in the correlation functions (22)-(25) to get 


$$
\begin{aligned}
\hat{\mathcal{X}}_{1}^{k j}(\tau) \bar{\sigma}_{p}(t-\tau)= & \frac{1}{Z_{p}} \sum_{\alpha, \beta} \hat{P}^{k} \bar{\sigma}_{p}(t-\tau) \\
& \times \hat{W}_{p c}^{\alpha \beta} \hat{C}^{j} \hat{W}_{c p}^{\beta \alpha} e^{-\mathcal{E}_{p}^{\alpha} / T} e^{-i\left(\omega_{k j}+\nu_{\alpha \beta}\right) \tau}, \\
\hat{\mathcal{X}}_{2}^{k j}(\tau) \bar{\sigma}_{c}(t-\tau)= & \frac{1}{Z_{c} \alpha, \beta} \hat{P}^{k} \hat{W}_{p c}^{\alpha \beta} \bar{\sigma}_{c}(t-\tau) \\
& \times \hat{C}^{j} \hat{W}_{c p}^{\beta \alpha} e^{-\mathcal{E}_{c}^{\beta} / T} e^{-i\left(\omega_{k j}+\nu_{\alpha \beta}\right) \tau},
\end{aligned}
$$

and

$$
\begin{aligned}
& \hat{\mathcal{X}}_{3}^{k j}(\tau) \bar{\sigma}_{p}(t-\tau)=\frac{1}{Z_{p}} \sum_{\alpha, \beta} \hat{W}_{c p}^{\beta \alpha} \hat{P}^{k} \bar{\sigma}_{p}(t-\tau) \\
& \times \hat{W}_{p c}^{\alpha \beta} \hat{C}^{j} e^{-\mathcal{E}_{p}^{\alpha} / T} e^{-i\left(\omega_{k j}+\nu_{\alpha \beta}\right) \tau}, \\
& \hat{\mathcal{X}}_{4}^{k j}(\tau) \bar{\sigma}_{c}(t-\tau)=\frac{1}{Z_{c}} \sum_{\alpha, \beta} \hat{W}_{c p}^{\beta \alpha} \hat{P}^{k} \hat{W}_{p c}^{\alpha \beta} \bar{\sigma}_{c}(t-\tau) \\
& \times \hat{C}^{j} e^{-\mathcal{E}_{c}^{\beta} / T} e^{-i\left(\omega_{k j}+\nu_{\alpha \beta}\right) \tau} .
\end{aligned}
$$

Here the superscripts $\alpha$ and $\beta$ label the eigenstates of the bath Hamiltonians $\hat{F}_{p}$ and $\hat{F}_{c}$, respectively, $\mathcal{E}_{p}^{\alpha}$ and $\mathcal{E}_{c}^{\beta}$ are their corresponding eigenvalues, while

$$
\nu_{\alpha \beta} \equiv \mathcal{E}_{p}^{\alpha}-\mathcal{E}_{c}^{\beta} .
$$

The correlation functions (22)-(25) are simply obtained from Eqs. (A4)-(A7) summing up indices, i.e.,

$$
\hat{\mathcal{J}}_{1-4}(\tau)=\sum_{k, j} \hat{\mathcal{X}}_{1-4}^{k j}(\tau) .
$$

Similarly, we fix the QDS transition frequency in the symmetrized correlation functions, which gives

$$
\hat{\mathcal{J}}_{1}^{k j}(\tau) \bar{\sigma}_{p}(t-\tau)=\sum_{\alpha, \beta} \hat{P}^{k} \bar{\sigma}_{p}(t-\tau) \hat{W}_{p c}^{\alpha \beta} \hat{C}^{j} \hat{V}_{c p}^{\beta \alpha} e^{-i\left(\omega_{k j}+\nu_{\alpha \beta}\right) \tau},
$$

$$
\hat{\mathcal{J}}_{2}^{k j}(\tau) \bar{\sigma}_{c}(t-\tau)=\sum_{\alpha, \beta} \hat{P}^{k} \hat{W}_{p c}^{\alpha \beta} \bar{\sigma}_{c}(t-\tau) \hat{C}^{j} \hat{V}_{c p}^{\beta \alpha} e^{-i\left(\omega_{k j}+\nu_{\alpha \beta}\right) \tau},
$$

and

$$
\begin{aligned}
& \hat{\mathcal{J}}_{3}^{k j}(\tau) \bar{\sigma}_{p}(t-\tau)=\sum_{\alpha, \beta} \hat{V}_{c p}^{\beta \alpha} \hat{P}^{k} \bar{\sigma}_{p}(t-\tau) \hat{W}_{p c}^{\alpha \beta} \hat{C}^{j} e^{-i\left(\omega_{k j}+\nu_{\alpha \beta}\right) \tau}, \\
& \hat{\mathcal{J}}_{4}^{k j}(\tau) \bar{\sigma}_{c}(t-\tau)=\sum_{\alpha, \beta} \hat{V}_{c p}^{\beta \alpha} \hat{P}^{k} \hat{W}_{p c}^{\alpha \beta} \bar{\sigma}_{c}(t-\tau) \hat{C}^{j} e^{-i\left(\omega_{k j}+\nu_{\alpha \beta}\right) \tau},
\end{aligned}
$$

where $\hat{V}_{c p}=\hat{V}_{c p}^{h}(0)$ was defined in Eq. (29). obtain

Taking the Fourier transform of Eqs. (A4) and (A10) we

$$
\begin{aligned}
& \hat{\tilde{\mathcal{X}}}_{1}^{k j}(\omega) \widetilde{\sigma}_{p}(\omega)=\frac{1}{Z_{p}} \sum_{\alpha, \beta} \hat{P}^{k} \widetilde{\sigma}_{p}(\omega) \hat{W}_{p c}^{\alpha \beta} \hat{C}^{j} \hat{W}_{c p}^{\beta \alpha} e^{-\mathcal{E}_{p}^{\alpha} / T} \\
& \times \delta\left(\omega+\omega_{k j}+\nu_{\alpha \beta}\right),
\end{aligned}
$$

and

$$
\begin{aligned}
\hat{\tilde{J}}_{1}^{k j}(\omega) \widetilde{\sigma}_{p}(\omega)= & \frac{1}{2} \sum_{\alpha, \beta} \hat{P}^{k} \widetilde{\sigma}_{p}(\omega) \hat{W}_{p c}^{\alpha \beta} \hat{C}^{j}\left(\frac{e^{-\mathcal{E}_{c}^{\beta} / T}}{Z_{c}} \hat{W}_{c p}^{\beta \alpha}\right. \\
& \left.+\hat{W}_{c p}^{\beta \alpha} \frac{e^{-\mathcal{E}_{p}^{\alpha} / T}}{Z_{p}}\right) \delta\left(\omega+\omega_{k j}+\nu_{\alpha \beta}\right) .
\end{aligned}
$$

Then we use the identity

$$
\begin{aligned}
\delta\left(\omega+\omega_{k j}+\nu_{\alpha \beta}\right) \frac{e^{-\mathcal{E}_{c}^{\beta} / T}}{Z_{c}} \equiv & \delta\left(\omega+\omega_{k j}+\nu_{\alpha \beta}\right) \\
& \times \frac{e^{-\mathcal{E}_{p}^{\alpha} / T}}{Z_{p}} e^{-\left(\omega+\omega_{k j}\right) / T} e^{-\epsilon_{p c} / T},
\end{aligned}
$$

where $\epsilon_{p c}$ is the change of the free energy in the course of reaction (35) to express the first term in the brackets on the right-hand side of Eq. (A15) through the second one, and, comparing the resulting expression with Eq. (14), we obtain

$$
\hat{\tilde{\mathcal{X}}}_{1}^{k j}(\omega) \widetilde{\sigma}_{p}(\omega)=\hat{\tilde{J}}_{1}^{k j}(\omega)\left(1-i \tilde{f}_{b}\left(\omega+\omega_{k j}+\epsilon_{p c}\right)\right) \widetilde{\sigma}_{p}(\omega),
$$

where

$$
\tilde{f}_{b}(\omega)=i \tanh \left(\frac{\omega}{2 T}\right) .
$$

The other relations are proved in the same way, which gives

$$
\begin{aligned}
& \hat{\tilde{\mathcal{X}}}_{1,3}^{k j}(\omega) \widetilde{\sigma}_{p}(\omega)=\hat{\tilde{\mathcal{J}}}_{1,3}^{k j}(\omega)\left(1-i \tilde{f}_{b}\left(\omega+\omega_{k j}+\epsilon_{p c}\right)\right) \widetilde{\sigma}_{p}(\omega), \\
& \hat{\tilde{\mathcal{X}}}_{2,4}^{k j}(\omega) \widetilde{\sigma}_{c}(\omega)=\hat{\tilde{\mathcal{J}}}_{2,4}^{k j}(\omega)\left(1+i \tilde{f}_{b}\left(\omega+\omega_{k j}+\epsilon_{p c}\right)\right) \widetilde{\sigma}_{c}(\omega) .
\end{aligned}
$$

The expressions (A19) and (A20) were obtained by identical transformations, no additional approximations were employed. Again, Eqs. (A19) and (A20) separate out the dynamical and the statistical properties of the heat reservoir, see also Ref. 40. The former enter via the symmetrized correlation functions $\hat{\mathcal{X}}_{1-4}$, which allow for the classical limit and contain all information about the dynamics of the bath. In turn, the statistical properties of the bath enter via universal (dynamics independent) function of the temperature, see Eq. (A18).

As it was found in Ref. 40, the generalized kernel (A18) describes the decay of quantum correlations and is in the time domain a sharply decaying function with the characteristic time $\tau_{b}$, Eq. (8), 


$$
f_{b}(t)=-\frac{1}{\pi \tau_{b}} \frac{1}{\sinh \left(t / \tau_{b}\right)} .
$$

Using the shortness of $\tau_{b}$ (it is just 8 fs at room temperature), one can integrate out of the quantum correlations contribution. The resulting kinetic equations are valid at times $t$ $\gg \tau_{b}$ and properly account for the detailed balance.

First, we use the identity

$$
\begin{aligned}
\widetilde{f}_{b}\left(\omega+\omega_{k j}+\epsilon_{p c}\right) \hat{P}^{k} \widetilde{\sigma}_{p}(\omega) \equiv & \int_{-\infty}^{\infty} e^{-i \omega t} \int_{-\infty}^{\infty} f_{b}(\tau) e^{-i\left(\omega_{k j}+\epsilon_{p c}\right) \tau} \\
& \times \hat{P}^{k} \bar{\sigma}_{p}(t-\tau) d \tau d t, \quad(\mathrm{~A} 22)
\end{aligned}
$$

and expand the density matrix $\bar{\sigma}_{p}$ (via the expansion of the density matrix in the interaction representation, see Ref. 40 for more details) as

$$
\bar{\sigma}_{p}(t-\tau) \underset{\tau \sim \tau_{b}}{\simeq} e^{i \hat{H}_{p} \tau}\left(\bar{\sigma}_{p}(t)-i\left[\hat{H}_{p}, \bar{\sigma}_{p}(t)\right] \tau-\frac{d \bar{\sigma}_{p}(t)}{d t} \tau\right) e^{-i \hat{H}_{p} \tau} .
$$

Then, substituting the expansion (A23) in the right-hand side of Eq. (A22), and replacing operator exponents by the relation (A2) we obtain

$$
\begin{gathered}
\tilde{f}_{b}\left(\omega+\omega_{k j}+\epsilon_{p c}\right) \hat{P}^{k} \widetilde{\sigma}_{p}(\omega) \simeq \sum_{n=1}^{N_{p}} \hat{P}^{k}\left(\widetilde{f}_{b}\left(\omega_{n j}+\epsilon_{p c}\right) \widetilde{\sigma}_{p}(\omega)\right. \\
\left.+\widetilde{h}_{b}\left(\omega_{n j}+\epsilon_{p c}\right)\left\{i \omega \widetilde{\sigma}_{p}(\omega)+i\left[\hat{H}_{p}, \widetilde{\sigma}_{p}(\omega)\right]\right\}\right) \hat{P}^{n},
\end{gathered}
$$

where

$$
\tilde{h}_{b}(\omega)=-\int_{-\infty}^{\infty} \tau f_{b}(\tau) e^{-i \omega \tau} d \tau=\frac{1}{2 T} \frac{1}{\cosh ^{2}\left(\frac{\omega}{2 T}\right)},
$$

and it follows from this that

$$
\begin{aligned}
\tilde{f}_{b}\left(\omega+\omega_{k j}+\epsilon_{p c}\right) \hat{P}^{k} \widetilde{\sigma}_{p}(\omega) \hat{W}_{p c} \hat{C}^{j} \\
\\
\simeq \hat{P}^{k}\left(\widetilde{\sigma}_{p}(\omega) \hat{U}_{p c}+\left\{i \omega \widetilde{\sigma}_{p}(\omega)+i\left[\hat{H}_{p}, \widetilde{\sigma}_{p}(\omega)\right]\right\} \hat{Y}_{p c}\right) \hat{C}^{j} .
\end{aligned}
$$

Here

$$
\begin{aligned}
& \hat{U}_{p c}=i \sum_{k, j} \tanh \left(\frac{\omega_{k j}+\epsilon_{p c}}{2 T}\right) \hat{P}^{k} \hat{W}_{p c} \hat{C}^{j}, \\
& \hat{Y}_{p c}=\frac{1}{2 T} \sum_{k, j} \frac{\hat{P}^{k} \hat{W}_{p c} \hat{C}^{j}}{\cosh ^{2}\left(\frac{\omega_{k j}+\epsilon_{p c}}{2 T}\right)},
\end{aligned}
$$

which can also be written as Eqs. (33) and (34). Similarly, we obtain

$$
\begin{aligned}
\tilde{f}_{b}\left(\omega+\omega_{k j}+\epsilon_{p c}\right) \hat{P}^{k} \hat{W}_{p c} \widetilde{\sigma}_{c}(\omega) \hat{C}^{j} \\
\simeq \hat{P}^{k}\left(\hat{U}_{p c} \widetilde{\sigma}_{c}(\omega)+\hat{Y}_{p c}\left\{i \omega \widetilde{\sigma}_{c}(\omega)+i\left[\hat{H}_{c}, \widetilde{\sigma}_{c}(\omega)\right]\right\}\right) \hat{C}^{j} .
\end{aligned}
$$

Finally, substituting Eqs. (A26) and (A29) into Eqs. (A19) and (A20), and taking the inverse Fourier transform, we derive

$$
\begin{aligned}
& -\hat{\tilde{\mathcal{X}}}_{1}^{k j}(\tau) \bar{\sigma}_{p}(t-\tau)+\hat{\tilde{\mathcal{X}}}_{2}^{k j}(\tau) \bar{\sigma}_{c}(t-\tau) \\
& =\sum_{\alpha, \beta} \hat{P}^{k} \bar{\gamma}_{p c}^{\alpha \beta}(t-\tau) \hat{C}^{j} i \hat{V}_{c p}^{\beta \alpha} e^{-i\left(\omega_{k j}+\nu_{\alpha \beta}\right) \tau},
\end{aligned}
$$

and

$$
\begin{aligned}
\hat{\tilde{\mathcal{X}}}_{3}^{k j}(\tau) & \bar{\sigma}_{p}(t-\tau)-\hat{\tilde{\mathcal{X}}}_{4}^{k j}(\tau) \bar{\sigma}_{c}(t-\tau) \\
& =-\sum_{\alpha, \beta} i \hat{V}_{c p}^{\beta \alpha} \hat{P}^{k} \bar{\gamma}_{p c}^{\alpha \beta}(t-\tau) \hat{C}^{j} e^{-i\left(\omega_{k j}+\nu_{\alpha \beta}\right) \tau},
\end{aligned}
$$

where $\bar{\gamma}_{p c}(t-\tau)$ is given by Eq. (32). Taking account for Eqs. (A9) and (A2) we obtain Eqs. (30) and (31).

\section{APPENDIX B: STATIONARY SOLUTION OF KINETIC EQUATIONS}

The stationary limit of the kinetic equations (45) and (46) requires $\eta_{p}(q, t)=\eta_{c}(q, t)=0$, and $\gamma_{p c}(q, t)=0$, see Eqs. (50) and (48). The first condition is satisfied by Eqs. (59), as it was already shown in Ref. 40, while the second one leads to the following relation to prove:

$$
\begin{aligned}
\alpha_{p} \sum_{k}^{N_{p}} \sum_{j}^{N_{c}}\left[1+\tanh \left(\frac{\omega_{k j}+\epsilon_{p c}}{2 T}\right)\right] e^{-E_{p}^{k} / T} \hat{P}^{k} \hat{W}_{p c}(q) \hat{C}^{j} \\
=\alpha_{c} \sum_{k}^{N_{p}} \sum_{j}^{N_{c}}\left[1-\tanh \left(\frac{\omega_{k j}+\epsilon_{p c}}{2 T}\right)\right] \hat{P}^{k} \hat{W}_{p c}(q) \hat{C}^{j} e^{-E_{c}^{j} / T},
\end{aligned}
$$

where we explicitly substituted Eq. (30), and used the identities

$$
e^{-\hat{H}_{p} / T} \hat{P}^{k} \equiv e^{-E_{p}^{k} / T} \hat{P}^{k}, \quad \hat{C}^{j} e^{-\hat{H}_{c} / T} \equiv \hat{C}^{j} e^{-E_{c}^{j} / T} .
$$

The relation (B1) holds for any term in the sum, i.e., at fixed indices $k, j$, if the condition (60) is true.

\footnotetext{
${ }^{1}$ See, e.g., articles in Encyclopedia of Chemical Physics and Physical Chemistry, Parts A and C, edited by J. H. Moore and N. D. Spencer (IoP, Bristol, 2001).

${ }^{2}$ Electron and Proton Transfer in Chemistry and Biology, Studies in Physical and Theoretical Chemistry, edited by A. Müller, Vol. 78, H. Ratajczak, W. Junge, and E. Diemann (Elsevier, Amsterdam, 1992).

${ }^{3}$ Proton Transfer in Hydrogen-Bonded Systems, edited by T. Bountis (Plenum, New York, 1993).

${ }^{4}$ J. March, Advanced Organic Chemistry. Reactions, Mechanisms, and Structure, 4th ed. (Wiley, New York, 1992).

${ }^{5}$ C. Reichardt, Solvent Effects in Organic Chemistry (Verlag Chemie, New York, 1979).

${ }^{6}$ The Hydrogen Bond: Recent Developments in Theory and Experiments, edited by P. Schuster, G. Zundel, and C. Sandorfy (North-Holland, Amsterdam, 1976), Vols. 1-3.

${ }^{7}$ B. E. Conway, Ionic Hydration in Chemistry and Biophysics (Elsevier, Amsterdam, 1981).

${ }^{8}$ Y. Marcus, Ion Solvation (Wiley, Chichester, 1985).

${ }^{9}$ W. N. Olmstead and J. I. Brauman, J. Am. Chem. Soc. 99, 4219 (1977).

${ }^{10}$ S. Efrima and M. Bixon, Chem. Phys. Lett. 25, 34 (1974).

${ }^{11}$ J. Jortner and M. Bixon, J. Chem. Phys. 88, 167 (1988).

${ }^{12}$ A. M. Kuznetsov, Charge Transfer in Physics, Chemistry, and Biology (Gordon and Breach, New York, 1995).

${ }^{13}$ M. Smoluchowski, Z. Phys. Chem., Stoechiom. Verwandtschaftsl. 92, 129
} (1917). 
${ }^{14}$ K. M. Hong and J. Noolandi, J. Chem. Phys. 68, 5163 (1978).

${ }^{15}$ A. I. Burshtein, Adv. Chem. Phys. 114, 419 (2000).

${ }^{16}$ A. A. Kipriyanov, A. B. Doktorov, and A. I. Burshtein, Chem. Phys. 76, 149 (1983); 76, 163 (1983).

${ }^{17}$ S. A. Rice, Diffusion-Limited Reactions (Elsevier, Amsterdam, 1985).

${ }^{18}$ J. Keizer, Chem. Rev. (Washington, D.C.) 87, 167 (1987).

${ }^{19}$ E. Kotomin and V. Kuzovkov, Modern Aspects of Diffusion-Controlled Reactions (Elsevier, Amsterdam, 1996).

${ }^{20}$ J. Sung and S. Lee, J. Chem. Phys. 111, 10159 (1999); 112, 2128 (2000).

${ }^{21}$ See, e.g., review articles published in Chem. Rev. (Washington, D.C.) 99 , 353 (1999).

${ }^{22}$ N. Makri, Annu. Rev. Phys. Chem. 50, 167 (1999), and references therein.

${ }^{23}$ M. P. Allen and D. J. Tildesley, Computer Simulation of Liquids (Clarendon, Oxford, 1996).

${ }^{24}$ P. A. M. Dirac, Proc. Cambridge Philos. Soc. 26, 376 (1930).

${ }^{25}$ G. D. Billing, J. Chem. Phys. 99, 5849 (1993).

${ }^{26}$ J. C. Tully and R. K. Preston, J. Chem. Phys. 55, 562 (1971).

${ }^{27}$ W. H. Miller and T. F. George, J. Chem. Phys. 56, 5637 (1972).

${ }^{28}$ F. Webster, J. Schnitker, M. S. Friedrich, R. A. Friesner, and P. J. Rossky, Phys. Rev. Lett. 66, 3172 (1991).

${ }^{29}$ P. J. Kunz, J. Chem. Phys. 95, 141 (1991).

${ }^{30}$ O. V. Prezhdo and P. J. Rossky, J. Chem. Phys. 107, 825 (1997).

${ }^{31}$ I. V. Alexandrov, Z. Naturforsch. A 36A, 902 (1981).
${ }^{32}$ W. Boucher and J. Traschen, Phys. Rev. D 37, 3522 (1988).

${ }^{33}$ A. Anderson, Phys. Rev. Lett. 74, 621 (1995).

${ }^{34}$ O. V. Prezhdo and V. V. Kisil, Phys. Rev. A 56, 162 (1997).

${ }^{35}$ R. Kapral and G. Cicotti, J. Chem. Phys. 110, 8919 (1999).

${ }^{36}$ M. Santer, U. Manthe, and G. Stock, J. Chem. Phys. 114, 2001 (2001).

${ }^{37}$ A. A. Neufeld, D. Schwarzer, J. Schroeder, and J. Troe, J. Chem. Phys. 119, 2502 (2003).

${ }^{38}$ P. A. Frantsuzov, Chem. Phys. Lett. 267, 427 (1997)

${ }^{39}$ P. A. Frantsuzov, J. Chem. Phys. 111, 2075 (1999)

${ }^{40}$ A. A. Neufeld, J. Chem. Phys. 119, 2488 (2003).

${ }^{41}$ B. F. Minaev, J. Mol. Struct.: THEOCHEM 183, 207 (1989).

${ }^{42}$ P. Suppan and N. Ghoneim, Solvatochromism (The Royal Society of Chemistry, Cambridge, 1997).

${ }^{43}$ S. Nakajima, Prog. Theor. Phys. 20, 948 (1958); R. Zwanzig, J. Chem. Phys. 33, 1338 (1960).

${ }^{44}$ A. A. Neufeld, J. Chem. Phys. 121, 2542 (2004).

${ }^{45}$ This question was raised by the referee.

${ }^{46}$ T. P. Straatsma and J. A. McCammon, Annu. Rev. Phys. Chem. 43, 407 (1992).

${ }^{47}$ W. F. van Gunsteren, X. Daura, and A. E. Mark, Helv. Chim. Acta 85, 3113 (2002).

${ }^{48}$ W. T. Pollard and R. A. Friesner, J. Chem. Phys. 100, 5054 (1994). 